\title{
TITLE:
}

\section{On topological Blaschke conjecture I: Cohomological complex projective spaces}

$\operatorname{AUTHOR}(\mathrm{S})$ :

SATO, Hajime

\section{CITATION:}

SATO, Hajime. On topological Blaschke conjecture I: Cohomological complex projective spaces. 数理解析研究所講究録 1983, 479: 77-92

\section{ISSUE DATE:}

1983-02

URL:

http://hdl.handle.net/2433/103362

RIGHT: 
On topological Blaschke conjecture I

Cohomological complex projective spaces

東比大理佐藤筆

(Hajime SATO)

By a Blaschke manifold, we mean a Riemannian manifold $(M, g)$ such that, for any point $m \in M$, the tangential cut locus $\mathrm{C}_{\mathrm{m}}$ of $\mathrm{m}$ in $\mathrm{T}_{\mathrm{m}} \mathrm{M}$ is isometric to the sphere of constant radius. There are some equivalent definitions (see Besse[2, 5.43]). The Blaschke conjecture is that any Blaschke manifold is isometric to a compact rank one symmetric space. If the integral cohomology $r i n g$ of $M$ is equal to the sphere $\mathrm{S}^{\mathrm{k}}$, or the real projective space $\mathrm{RP}^{\mathrm{k}}$, this conjecture is proved by Berger with other mathematiciens [2, Appendix D]). We consider the case where the cohomology ring of $M$ is equal to that of the complex projective space $\mathrm{CP}$.

We obtain the following theorem.

Theorem. Let $(M, g)$ be a $2 \mathrm{k}$-dimensional Blaschke manifold such that the integral cohomology ring is equal to that of $\mathrm{CP}^{\mathrm{k}}$. Then $\mathrm{M}$ is $\mathrm{PL}$-homeomorphic to $\mathrm{CP}^{\mathrm{k}}$ for any $k$.

Blaschke manifolds with other cohomology rings will be treated in subsequent papers. 
If $(M, g)$ is a Blaschke manifold and $m \in M$, Allamigeon [1] has shown that the cut locus $C(m)$ of $m$ in $M$ is the base manifold of a fibration of the tangential cut locus $C_{m}$ by great spheres. We study the base manifold of such fibration by great circles. We apply the BrowderNovikov-Sullivan's theory in the classification of homotopy equivalent manifolds (see Wall[4]). Calculation of normal invariants gives our theorem. In Appendix, we give examples of non-trivial fibrations of $\mathrm{s}^{3}$ by great circles. The author thanks to M.Mizutani and K.Masuda for the discussion of results in Appendix.

Detailed proof will appear elsewhere. 


\section{9}

§1. Projectable bundles

In the paper [3], we have obtained a method of a calculation of the tangent bundle of the base space of an $\mathrm{S}^{1}$-principal bundle. We will briefly recall that.

Let $\mathrm{X}$ be a smooth manifold and let $\pi: L, \rightarrow \mathrm{X}$ be the projection of an $S^{1}$-principal bundle.

Definition. A vector bundle $\mathrm{p}: \mathrm{E} \longrightarrow \mathrm{L}$ over $\mathrm{L}$ is projectable onto $\mathrm{x}$, if there exists a vector bundle $\hat{\mathrm{P}}: \hat{\mathrm{E}} \rightarrow \mathrm{X}$ over $\mathrm{X}$ such that $\pi * \hat{\mathrm{E}}=\mathrm{E}$. The map $\pi$ induces the bundle map $\pi_{!}: \mathrm{E} \longrightarrow \hat{\mathrm{E}}$, which we call the projection. The bundle $\hat{E}$ is called the projected bundle. Let $\mathrm{x}$ be $\mathrm{a}$ point in $\mathrm{x}$. For any $\mathrm{a}, \mathrm{b} \in \pi^{-1}(\mathrm{x})=\mathrm{s}^{1}$, we have a linear isomorphism

$$
\Phi_{\mathrm{ab}}: \mathrm{p}^{-1}(\mathrm{a}) \longrightarrow \mathrm{p}^{-1}(\mathrm{~b})
$$

of vector spaces defined by $\Phi_{a b}(u)=v$, where $\pi_{!}(u)=\pi_{!}(v)$. Then we have, for $a, b, c \pi^{-1}(x)$,

$$
\Phi_{\mathrm{bc}} \Phi_{\mathrm{ab}}=\Phi_{\mathrm{ac}}
$$

Let $\pi^{*} L=\{(a, b) \in I \times L, \pi(a)=\pi(b)\}$ be the induced $s^{1}$-bundle over $L$ from $L$. We have two projections $\pi_{1}$, $\pi_{2}: \pi^{*} \mathrm{~L} \longrightarrow$ L defined by $\pi_{1}(\mathrm{a}, \mathrm{b})=\mathrm{a}$ and $\pi_{2}(\mathrm{a}, \mathrm{b})=\mathrm{b}$. let $\pi_{i}{ }^{*} \quad(i=1,2)$ be the induced vector bundle. The map $\Phi: \pi^{*} \mathrm{~L} \longrightarrow \operatorname{Iso}\left(\pi_{1}{ }^{*} \mathrm{E}, \pi_{2}{ }^{*} \mathrm{E}\right)$ defined by $\Phi(\mathrm{a}, \mathrm{b})=\Phi_{\mathrm{ab}}$ is a continuous cross section of the bundle Iso $\left(\pi_{1}{ }^{*} \mathrm{E}, \pi_{2}{ }^{*} \mathrm{E}\right)$ over $\pi^{*} \mathrm{~L}$. 
We call $\Phi$ the projecting isomorphism associated with the projectable bundle $\mathrm{E}$.

Proposition 1. Suppose given a vector bundle E . over $L$ and a cross section $\Phi$ of the bundle Iso $\left(\pi_{1}{ }^{*} E, \pi_{2}{ }^{*} E\right)$ satisfying (1). Then we have a vector bundle $\hat{E}$ over $X$ such that $\pi^{*} \hat{E}=E$ and the projecting isomorphism is equal to $\Phi$.

Now let $T L$ and $T X$ be the tangent bundles of $L$ and $\mathrm{x}$ respectively. Let $\rho: s^{1} \times \mathrm{L} \longrightarrow \mathrm{L}$ be the free $s^{1}$-action. For each $t \in s^{1}$, the diffeomorphism $\rho(t)$ $=\rho(t,$.$) induces a budle isomorphism \rho(t)_{*}: T L \longrightarrow T L$. Proposition 2. The collection $\bigcup_{t \in S^{1}}^{\prime} \rho(t) *$ induces a projecting isomorphism on the bundle TL such that the projected bundle $\mathrm{TL}$ is isomorphic to $\mathrm{TX} \oplus 1$.

Proof. Choose a bundle metric on $\mathrm{TL}$. Let $\mathrm{TL}_{1}$ be the subnundle of TL consisting of tangent vectors normal to the $\mathrm{S}^{1}$-action. Then $\mathrm{TL}_{1}$ is projected to $\mathrm{TX}$. The line bundle tangent to the $S^{1}$-action is projected to the trivial line bundle on $\mathrm{x}$. 
§2. Pontrjagin classes

Let $\mathrm{s}^{2 \mathrm{k}-1}$ be the unit sphere in $\mathbb{R}^{2 \mathrm{k}}$ and let $\pi$ : $\mathrm{s}^{2 \mathrm{k}-1} \longrightarrow \mathrm{B}$ be a fibration of $\mathrm{s}^{2 \mathrm{k}-1}$ by great circles. Thus, for each $b \in B, \pi^{-1}(b)$ is the intersection of $\mathrm{s}^{2 \mathrm{k}-1}$ with a 2-plane in $\mathbb{R}^{2 \mathrm{k}}$. We write the 2-plane by $\mathrm{P}(\mathrm{b})$. Let $\rho: \mathrm{s}^{1} \times \mathrm{s}^{2 \mathrm{k}-1} \longrightarrow \mathrm{s}^{2 \mathrm{k}-1}$ denote the free $\mathrm{s}^{1}-$ action.

Let $V(2 k, 2)$ and $G(2 k, 2)$, respectively, be the Stiefel and the Grassmann manifold consisting of orthogonal 2 frames or oriented 2-planes in $\mathbb{R}^{2 \mathrm{k}}$. Then the natural mapping $\lambda: \mathrm{V}(2 \mathrm{k}, 2) \longrightarrow \mathrm{G}(2 \mathrm{k}, 2)$ defines a principal $\mathrm{s}^{1}$-bundle.

The mapping $\theta: B \longrightarrow G(2 k, 2)$ defined by $\theta(b)=$ $P(b)$ is a smooth embedding. Let $\theta *(\lambda)$ denote the induced bundle of $\lambda$ by $\theta$. Since $\pi$ is also the induced bundle of $\lambda$ by $\theta$, there exists a natural bundle isomorphism between $\pi$ and $\theta *(\lambda)$ inducing the identity on B. Thus we obtain;

Lemma 3. We may suppose that the free $S^{1}$-action $\rho$ on $\mathrm{s}^{2 \mathrm{k}-1}$ is equal to the restriction on $\pi^{-1}(b)$ of the linear action on $P(b)$ for every $b \in B$.

In the following, we always assume that $\rho$ is the linear action on each fibre. For each $x \in \mathrm{s}^{2 \mathrm{k}-1}$, let $\mathrm{Kx}$ denote the point $\rho(1 / 4) x$ in $s^{2 k-1}$, where we identify 


\section{2}

$\mathrm{S}^{1}$ with $[0,1] /[0] \sim[1]$. Define a mapping $\tilde{\theta}$ : $s^{2 k-1} \longrightarrow v(2 k, 2)$ by $\quad \tilde{\theta}(x)=(x, k x)$. This is a smooth embedding and is a bundle map inducing $\theta$ on the base manifolds. For an orthogonal 2-frame $\mathrm{w}=(\mathrm{x}, \mathrm{y})$, let $\Psi(\mathrm{w})$ denote the vector $(\mathrm{x} / \sqrt{2}, \mathrm{y} / \sqrt{2})$ in $\mathbb{R}^{2 \mathrm{k}} \oplus \mathbb{R}^{2 \mathrm{k}}$. Then the map $\psi: V(2 \mathrm{k}, 2) \longrightarrow \mathbb{R}^{4 \mathrm{k}}$ is a smooth embedding of $\mathrm{V}(2 \mathrm{k}, 2)$ in $\mathrm{s}^{4 \mathrm{k}-1} \subset \mathbb{R}^{4 \mathrm{k}}$. We identify $\mathbb{R}^{2 \mathrm{k}} \oplus \mathbb{R}^{2 \mathrm{k}}$ with $\mathbb{C}^{2 \mathrm{k}}$ such that the first summand $\mathbb{R}^{2 \mathrm{k}}$ is the real part and the second pure imaginary. On $\mathbb{C}^{2 \mathrm{k}}-0$, we have the free action $\rho_{0}$ of $S^{1}$ as the multiplication by the complex number of norm one. Then $\Psi$ is $\mathrm{S}^{1}$ - equivalent and we write by $\psi$ the induced map $\psi$ : $\mathrm{G}(2 \mathrm{k}, 2) \longrightarrow \mathbb{C P} \mathrm{P}^{2 \mathrm{k}-1}$.

Let $\mathrm{f}: \mathrm{s}^{2 \mathrm{k}-1} \longrightarrow \mathrm{s}^{4 \mathrm{k}-1}$ be the composition $\mathrm{f}=\tau \theta$ and $f=\psi \theta: B \longrightarrow \mathbb{C}^{2 \mathrm{k}-1}$. The map $\mathrm{f}$ is given by $f(x)=(x / \sqrt{2}, K x / \sqrt{2})$ for $x \in s^{2 k-1}$. We define a map $\tilde{F}: \mathbb{R}^{2 \mathrm{k}}-0 \longrightarrow \mathbb{C}^{2 \mathrm{k}}-0$ by $\tilde{F}(t x)=t \tilde{f}(x)$ for $t>0$ and $x \in s^{2 k-1}$. The map $\tilde{F}$ is a smooth embedding. Let $\mathrm{E}$ denote the restriction of the tangent bundle $T\left(\mathbb{R}^{2 \mathrm{k}}-0\right)$ of $\mathbb{R}^{2 \mathrm{k}}-0$ on $\mathrm{s}^{2 \mathrm{k}-1}$, and we write $\mathrm{p}$ for the projection $\mathrm{E} \longrightarrow \mathrm{S}^{2 \mathrm{k}-1}$. Then $\tilde{\mathrm{F}}$ induces an injective bundle map $\tilde{\mathrm{F}}_{*}: \mathrm{E} \longrightarrow \tilde{\mathrm{F}}_{*}(\mathrm{E}) \subset$ $T\left(\mathbb{C}^{2 k}-0\right) / \tilde{F}\left(S^{2 k-1}\right)$.

Now define a map $\tilde{G}: \mathbb{R}^{2 k}-0 \longrightarrow \mathbb{C}^{2 k}-0$ by $\tilde{G}(t x)=(t x / \sqrt{2},-t k / \sqrt{2})$ for $t>0$ and $x \in s^{2 k-1}$. 
Then $\tilde{G}$ is also an embedding and $\tilde{G}$ induces an injective bundle map

$$
G_{*}: E \rightarrow \widetilde{G}_{*}(E) \subset T\left(\mathbb{C}^{2 k-0}-0\right) \mid \tilde{G}\left(S^{2 k-1}\right) .
$$

If $\bar{\rho}_{0}$ denote the conjugate action of $s^{l}$ on $\mathbb{C}^{2 k}-0$. Then $G$ is $S^{1}$-equivariant concerning to this conjugate action.

For any $\mathrm{y} \in \mathbb{C}^{2 \mathrm{k}}$, we naturally identify the tangent space $T_{y} \mathbb{C}^{2 \mathrm{k}}$ with $\mathbb{C}^{2 \mathrm{k}}$ itself. For $\mathrm{x} \in \mathrm{S}^{2 \mathrm{k}-1}$, let $\mathrm{E}_{\mathrm{x}}$ denote the fiber $\mathrm{p}^{-1}(\mathrm{x})$. Then $\tilde{\mathrm{F}}_{*}\left(\mathrm{E}_{\mathrm{x}}\right)$ and $\tilde{\mathrm{G}}_{*}\left(\mathrm{E}_{\mathrm{x}}\right)$ are subvector spaces of $\mathbb{C}^{2 \mathrm{k}}$.

Since $K: s^{2 k-1} \longrightarrow s^{2 k-1}$ is a diffeomorphism, we obtain;

Lemma 4. The vector spaces $\tilde{\mathrm{F}}_{*}\left(\mathrm{E}_{\mathrm{x}}\right)$ and $\tilde{\mathrm{G}}_{*}\left(\mathrm{E}_{\mathrm{x}}\right)$ are transversal. Thus they span $\mathbb{C}^{2 \mathrm{k}}$.

Let $T$ denote the restriction of the tangent bundle $\mathrm{T}\left(\mathbb{C}^{2 \mathrm{k}}\right)$ on $\tilde{\mathrm{F}}\left(\mathrm{S}^{2 \mathrm{k}-1}\right)$. Then we have the direct sum decomposition by trivial vector bundles

$$
\mathrm{T}=\tilde{\mathrm{F}}_{*}(\mathrm{E}) \oplus \tilde{\mathrm{G}}_{*}(\mathrm{E})
$$

Notice that $\tilde{G}_{*}(E)$ on $\tilde{G}\left(S^{2 k-1}\right)$ is identified with the subbundle in $\mathrm{T}$ over $\tilde{\mathrm{F}}\left(\mathrm{S}^{2 \mathrm{k}-1}\right)$ by an orientation reversing diffeomorphism of $\mathrm{s}^{2 \mathrm{k}-1}$.

For any $t \in s^{1}$, we have the induced bundle isomorphisms $\rho_{*}(t): E \longrightarrow E$ and $\rho_{0 *}(t): T \longrightarrow T$. 
Lemma 5. The isomorphism $\rho_{0 *}(t)$ is equal to the direct sum

$$
\rho_{*}(t)+\rho_{*}(t)
$$

By Proposition l, we ob.tain $\hat{T}$, defined by the project-
that the projected bundle ing isomorphism $\rho_{*}(t)$, is isomorphic to the Whitney sum;

$$
\hat{\mathrm{T}} \cong \hat{\mathrm{E}} \oplus \hat{\mathrm{E}} \text {. }
$$

On the other hand, by Proposition ob'tain the following.

Lemma 6. The bundle $\hat{\mathrm{T}}$ has the complex structure. As a complex vector bundle, $\hat{\mathrm{T}}$ is isomorphic to the Whitney $\left.\operatorname{sum} T\left(\mathbb{C P} \mathrm{P}^{2 \mathrm{k}-1}\right)\right|_{\mathrm{f}(\mathrm{B})} \oplus 1$.

Lemma 7. As a real vector bundle, $\hat{\mathrm{E}}$ is isomorphic to the bundle $\mathrm{T}(\mathrm{B}) \oplus 2$.

Consequently, we obtain that

$$
\mathrm{T}(\mathrm{B}) \oplus \mathrm{T}(\mathrm{B}) \oplus 4 \cong\left(\left.\mathrm{T}\left(\mathbb{C P}^{2 \mathrm{k}-1}\right)\right|_{\mathrm{f}(\mathrm{B})} \oplus 1\right)_{\mathbb{R}} .
$$

Since the cohomology groups $H^{*}(B ; \mathbb{Z})$ has no torsion element, by the product formula of Pontrjagin classes, we obtain the following.

Proposition 8. The Pontrjagin classes of the smooth manifold $B$ is equal to that of $\mathbb{C P} \mathrm{P}^{\mathrm{k}-1}$, for any $\mathrm{k}$. 
§3. $\mathrm{z}_{2}$-invariants and proof of Theorem

Let $\&\left(\mathrm{CP}^{\mathrm{k}-1}\right)$ denote the set of $\mathrm{PL}$-homeomorphism classes of closed PL-manifolds homotopy equivalent to $\mathrm{CP}^{\mathrm{k}-1}$. The following results are due to sullivan (cf. $[4, \S 14 \mathrm{C}])$.

\section{Suppose that $k>3$.}

Proposition 9. For any $\mathrm{N} \in \&\left(\mathrm{CP}^{\mathrm{k}-1}\right)$, there are invariants $\mathrm{s}_{4 i+2}(\mathrm{~N}) \in \mathrm{z}_{2}$ and $\mathrm{s}_{4 j}(\mathrm{~N}) \in \mathrm{z}$, for all integers $i, j$ satisfying $6 \leq 4 i+2<2(k-1)$, $4 \leqslant 4 j<2(k-1)$. The invariants define a bijection of $\varnothing\left(\mathrm{CP}^{\mathrm{k}-1}\right) \quad$ with

$$
\underset{i}{\left(\oplus z_{2}\right) \oplus(\oplus z)}
$$

The invariants $s_{4 j}$ satisfy the following relations.

Proposition 10. If all the Pontrjagin classes of $\mathrm{N}$ in $f\left(\mathrm{CP}^{\mathrm{k}-1}\right)$ coincide with that of $\mathrm{CP}^{\mathrm{k}-1}$, then $\mathbf{s}_{4 j}(\mathrm{~N})=0$ for all $j$.

\section{Concerning $\mathrm{z}_{2}$-invariants $\mathrm{s}_{4 i+2}$, the following} holds. Let $\&\left(\mathrm{RP}^{2 \mathrm{k}-1}\right)$ denote the set of $\mathrm{PL}$-homeomorphism classes of closed PL-manifolds homotopy equivalent to $\mathrm{RP}^{2 \mathrm{k}-1}$. This set is known to be equal to the isomorphism classes of homotopy triangulations of $\mathrm{RP}^{2 \mathrm{k}-1}$. Any $\mathrm{N}$ $\epsilon \varnothing\left(\mathrm{CP}^{\mathrm{k}-1}\right)$ is the base manifold of a PL free $\mathrm{s}^{1}$-action on $\mathrm{s}^{2 \mathrm{k}-1}$. By restricting the action to $\mathrm{z}_{2}=\mathrm{s}^{0} \subset \mathrm{s}^{1}$, we obtain a manifold homotopy equivalent to $\mathrm{RP}^{2 \mathrm{k}-1}$. 
This defines a map

$$
\pi^{\mathrm{b}}: \not\left(\mathrm{CP}^{\mathrm{k}-1}\right) \longrightarrow \not\left(\mathrm{RP}^{2 \mathrm{k}-1}\right)
$$

The following holds ([4, §l4D.3]).

Proposition 11. Let $\mathrm{N}$ be an element in $\&\left(\mathrm{CP}^{\mathrm{k}-1}\right)$ such that $\pi^{\mathrm{b}}(\mathrm{N})$ is $\mathrm{PL}$-homeomorphic to $\mathrm{RP}^{2 \mathrm{k}-1}$. Then

$$
\mathbf{s}_{4 i+2}(\mathrm{~N})=0,
$$

for all $i$.

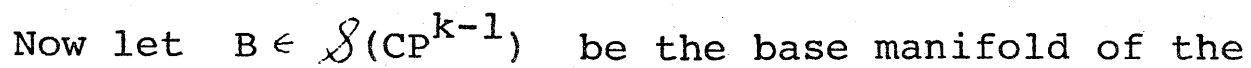
fibration of $\mathrm{s}^{2 \mathrm{k}-1}$ by great circles. Then, obviously, the image $\pi^{\mathrm{b}}(\mathrm{B}) \in \varnothing\left(\mathrm{RP}^{2 \mathrm{k}-1}\right)$ is $\mathrm{PL}$-homeomorphic to RP $2 \mathrm{k}-1$.

Combining the result of $\S 2$ with Propositions, we obtain ;

Proposition 12. The base manifold $B$ of a fibration of $\mathrm{s}^{2 \mathrm{k}-1}$ by great circles is $\mathrm{PL}$-homeomorphic to $\mathrm{CP}^{\mathrm{k}-1}$ if $\mathrm{k} \neq 3$.

Now let us prove Theorem. Since the integral cohomology ring of $M$ is equal to that of $C P^{k}, M$ is simply connected $([2,7.23])$. Thus $M$ is homotopy equivalent to $\mathrm{CP}^{\mathrm{k}}$. By Allamigeon's theorem, we know that $M$ is $P L$-homeomorphic to the union of the disc $D^{2 k}$ with the $D^{2}$-bundle associated with the fibration of $s^{2 k-1}$ by great circles. We write. B. for the base manifold of the fibration. If $k=3$, by Proposition 9, $M$ is 
PL-homeomorphic to $\mathrm{CP}^{3}$ if and only if $\mathrm{s}_{4}(\mathrm{M})=0$. The invariant $\mathrm{s}_{4}(\mathrm{M})$ is calculated from the first Pontrjagin class $p_{1}(B)$ of $B$. By Proposition 8 of $\S 2$, $\mathrm{p}_{1}(\mathrm{~B})$ is equal to $\mathrm{p}_{1}\left(\mathrm{CP}^{2}\right)$. Thus we have $\mathrm{s}_{4}(\mathrm{M})=0$ and $M$ is $\mathrm{PL}$-homeomorphic to $\mathrm{CP}^{3}$. Now suppose that $\mathrm{k} \neq 3$. According to Proposition 12, $\mathrm{B}$ is PLhomeomorphic to $\mathrm{CP}^{\mathrm{k}-1}$. The Euler class of the $\mathrm{s}^{1}-$ bundle is equal to a generator of $\mathrm{H}^{2}\left(\mathrm{CP}^{\mathrm{k}-1} ; \mathrm{Z}\right)=\mathrm{Z}$. Thus the total space of the $\mathrm{D}^{2}$-bundle is $\mathrm{PL}$-homeomorphic to the tubular neighborhood of $\mathrm{CP}^{\mathrm{k}-1}$ in $\mathrm{CP}^{\mathrm{k}}$. Any orientation preserving PL-homeomorphism of $\mathrm{s}^{2 \mathrm{k}-1}$ is isotopic to the identity. The attached manifold $M$ is $\mathrm{PL}$-homeomorphic to $\mathrm{CP}^{\mathrm{k}}$, which completes the proof of Theorem. 
§4. Appendix

If $\pi: \mathrm{s}^{2 \mathrm{k}-1} \longrightarrow \mathrm{B}$ is a fibration by great circles, we get the embedding $\theta: B \longrightarrow G(2 k, 2)$. Since the planes $\theta(b)$ for $a l l b \in B$ give a foliation of $s^{2 k-1}$, we have the following property.

(*) For two different points $b$ and $b^{\prime}$ in $B$, the planes $\theta(b)$ and $\theta\left(b^{\prime}\right)$ are transverse.

The converse holds.

Lemma 13. Let $\pi: \mathrm{s}^{2 \mathrm{k}-1} \longrightarrow \mathrm{B}$ be a principal $\mathrm{s}^{1}-$ bundle induced from the $S^{l}$-bundle $\lambda: W(2 k, 2) \longrightarrow G(2 k, 2)$ by a smooth embedding $\theta: B \longrightarrow G(2 k, 2)$. Suppose that, for any different points $b$ and $b^{\prime}$ in $B$, the planes $\theta(b)$ and $\theta\left(b^{\prime}\right)$ are transversal. Then the bundle $\pi$ is a fibration of $\mathrm{s}^{2 \mathrm{k}-1}$ by great circles.

Proof. Consider the union $\bigcup_{b}^{\prime}\left(\theta(b) \cap s^{2 k-1}\right)$. Then it covers $\mathrm{s}^{2 \mathrm{k}-1}$ and give a fibration by great circles. Now we consider the case where $k=2$. For the following discussion, see $[2, \mathrm{p} .55]$. Let $\Lambda^{2} \mathbb{R}^{4}$ denote the space of skew-symmetric 2-tensors. The Grassmann manifold $G(4,2)$ is naturally identified with the set of decomposable elements of norm one in $\Lambda^{2} \mathbb{R}^{4}$. We have the Hodge operator * from $\Lambda^{2} \mathbb{R}{ }^{4}$ onto itself. The space $\Lambda^{2} \mathbb{R}^{4}$ is decomposed to two orthogonal subsets $E_{1}$ and $E_{-1}$ associated to the eigenvalue 1 and -1 of * . 
let $\mathrm{s}_{1}^{2}$ and $\mathrm{s}_{-1}^{2}$ be the sphere in $E_{1}$ and $E_{-1}$ of radius $1 / \sqrt{2}$. Then $G(4,2)$ is equal to the product $\mathrm{S}_{1}^{2} \times \mathrm{s}_{-1}^{2}$. Define a bilenear map $\zeta: \Lambda^{2} \mathbb{R}^{4} \times \Lambda^{2} \mathbb{R}^{4}$ $\longrightarrow \mathbb{R}$ by $\zeta(a, b)=\left\|a_{\Lambda} b\right\|$, where \|\| is the norm on $\Lambda^{2} \mathbb{R}^{4} \cong \mathbb{R}$. Two planes $P_{1}$ and $P_{2}$ in $G(4,2)$ are transversal if and only if $\zeta\left(\mathrm{P}_{1}, \mathrm{P}_{2}\right)=0$. Represent $\mathrm{P}_{1}$ and $\mathrm{P}_{2}$ by $\left(\mathrm{x}_{1}, \mathrm{x}_{2}\right)$ and $\left(\mathrm{y}_{1}, \mathrm{y}_{2}\right)$, where $\mathrm{x}_{1}, \mathrm{y}_{1}$ $\in s_{1}^{2}$ and $x_{2}, y_{2} \in s_{-1}^{2}$. Then we have

$$
\zeta\left(\mathrm{P}_{1}, \mathrm{P}_{2}\right)=\left\langle\mathrm{x}_{1}, \mathrm{y}_{1}\right\rangle-\left\langle\mathrm{x}_{2}, \mathrm{y}_{2}\right\rangle,
$$

where \langle\rangle is the inner product of the vector space $E_{1}$ or $E_{-1}$.

For a smooth map $\theta: s^{2} \longrightarrow G(4,2)$, we define a smooth function $z(\theta)$ on $S^{2}$ by $z(\theta)(x)=\zeta\left(\theta(x), \theta\left(x^{\prime}\right)\right)$, by fixing $x^{\prime}$ in $s^{2}$. Thus the principal $s^{1}$-bundle $\pi$ : $\mathrm{s}^{3} \longrightarrow \mathrm{s}^{2}$ induced by an embedding $\theta: \mathrm{s}^{2} \longrightarrow \mathrm{G}(4,2)$ is a fibration by great circles if $\mathrm{z}(\theta)(\mathrm{x})=0$ only when $\mathrm{x}=\mathrm{x}^{\prime}$. Obviously $\mathrm{z}(\theta)(\mathrm{x})=0$ at $\mathrm{x}=\mathrm{x}^{\prime}$. We have;

Lemma 14. For a smooth map $\theta: s^{2} \longrightarrow G(4,2)$, the function $\mathrm{z}(\theta)$, for fixed $\mathrm{x}^{\prime} \in \mathrm{S}^{2}$, is critical at $\mathrm{x}=\mathrm{x}^{\prime}$.

Proof. Fix $\mathrm{P}_{2}$ in $\mathrm{G}(4,2)$. The function $\zeta\left(\mathrm{P}_{1}, \mathrm{P}_{2}\right)$ on $G(4,2)$ is critical at $P_{1}=P_{2}$. Thus $Z(\theta)$ is also critical at $\mathrm{x}=\mathrm{x}^{\prime}$.

Now consider the Hopf fibration $\pi_{0}: s^{3} \longrightarrow s^{2}$. 
The associated map ${ }^{\theta} 0: s^{2} \longrightarrow G(4,2)=s^{2}{ }_{1} \times s^{2}-1$ is given by $\theta_{0}(x)=\left(1 / \sqrt{2} x, \alpha_{0}\right)$, where $\alpha_{0}=$ $(1 / \sqrt{2}, 0,0)$. For two points $\mathrm{x}=\left(\mathrm{x}_{1}, \mathrm{x}_{2}, \mathrm{x}_{3}\right)$ and $x^{\prime}=\left(x_{1}^{\prime}, x_{2}^{\prime}, x_{3}^{\prime}\right)$ in $s^{2}$, we have

$$
\begin{aligned}
\zeta\left(\theta_{0}(x), \theta_{0}\left(x^{\prime}\right)\right) & =\left\langle x, x^{\prime}\right\rangle-1 / 2 \\
& =-1 / 2 \sum\left(x_{i}-x_{i}^{\prime}\right)^{2} .
\end{aligned}
$$

Thus the function $\mathrm{z}\left(\theta_{0}\right)$ is critical if and only if $\mathrm{x}$ $=x^{\prime}$. The symmetric matrix $\left(\partial^{2} z\left(\theta_{0}\right) / \partial x_{i} \partial x_{j}\right)$ is positive definite.

Let $\operatorname{Emb}\left(s^{2}, G(4,2)\right)$ denote the set of smooth embeddings of $s^{2}$ in $G(4,2)$ with $C^{2}$-topology. Since $\mathrm{s}^{2}$ is compact, we obtain the following.

Proposition 15. There exists a neighborhood $\mathrm{U}$ of $\theta_{0}$ in $\operatorname{Emb}\left(s^{2}, G(4,2)\right)$ such that the function $Z(\theta)\left(x, x^{\prime}\right)$ $=\zeta\left(\theta(x), \theta\left(x^{\prime}\right)\right)$ is equal to zero if and only if $x=x^{\prime}$, for any $\mathrm{x}, \mathrm{x}^{\prime} \in \mathrm{s}^{2}$ and $\theta \in \mathrm{U}$.

Corollary 16. In each direction in $\operatorname{Emb}\left(\mathrm{s}^{2}, \mathrm{G}(4,2)\right)$, there is a deformation of fibrations of $s^{3}$ by great circles starting from the Hopf fibration. 
The group of diffeomorphisms of $s^{2}$, denoted by Diff $S^{2}$, acts naturally on $\operatorname{Emb}\left(S^{2}, G(4,2)\right)$. We denote by $\operatorname{Diff} S^{2} \backslash \mathrm{Emb}\left(S^{2}, G(4,2)\right)$ the quotient space. Let $\pi: S^{3} \longrightarrow B$ be a fibration of $S^{3}$ by great circles. The $B$ is diffeomorphic to $\mathrm{s}^{2}$. Thus we have the class $\{\theta\}$ in $\operatorname{Diff} S^{2} \backslash \operatorname{Emb}\left(S^{2}, G(4,2)\right)$.

Let $\pi_{1}$ and $\pi_{2}$ be two fibrations of $s^{3}$ by great circles, and let $\left\{\theta_{1}\right\},\left\{\theta_{2}\right\} \in \operatorname{Diff} S^{2} \backslash \operatorname{Emb}\left(S^{2}, G(4,2)\right)$ be the associated classes. We say that $\pi_{1}$ and $\pi_{2}$ are isometric if there exists a budle map $F$ from $\pi$ to $\pi_{2}$ such that $F$ is an isometry of $S^{3}$ onto itself. The group $O(4)$ acts naturally on $G(4,2)$ and on $\operatorname{Diff} S^{2} \backslash \operatorname{Emb}\left(S^{2}, G(4,2)\right)$. We denote by $\operatorname{Diff} S^{2} \backslash \operatorname{Emb}\left(S^{2}, G(4,2)\right) / O(4)$ the quotient space.

Proposition 17. Two fibrations $\pi_{1}$ and $\pi_{2}$ of $\mathrm{s}^{3}$ by great circles are isometric if and only if the classes $\left\{\theta_{1}\right\}$ and $\left\{\theta_{2}\right\}$ in $\operatorname{Diff} S^{2} \backslash \operatorname{Emb}\left(S^{2}, G(4,2)\right) / O(4)$ are equal.

Remark that we can choose the neighborhood $U$ in Proposition 15 such that $U$ is invariant by the actions of Diff $\mathrm{s}^{2}$ and $\mathrm{O}(4)$. The space Diff $\mathrm{s}^{2} \backslash \mathrm{U} / \mathrm{O}(4)$ is of infinite"dimension". 
REFERENCES

[1] A.Allamigeon, Propriété globales des espaces de Riemann harmoniques, Ann. Inst.Fourier, 15 (1965), $91-132$.

[2] A. Besse, Manifolds all of whose geodesics are closed, Ergebnisse der Math., 93 (1978), Springer.

[3] H.Sato, On the manifolds of periodic geodesics, this volume.

[4] C.T.C.Wall, Surgery on compact manifolds, Academic press (1970), London. 